\title{
Human in vivo fluorescence microimaging of the alveolar ducts and sacs during
} bronchoscopy

\author{
L. Thiberville*, , M. Salaün*,*, S. Lachkar*, S. Dominique*, S. Moreno-Swirc*, \\ C. Vever-Bizet and G. Bourg-Heckly"
}

ABSTRACT: The aim of the present study was to assess fibred confocal fluorescence microscopy (FCFM) as a tool for imaging the alveolar respiratory system in vivo during bronchoscopy.

A 488-nm excitation wavelength FCFM device was used in 41 healthy subjects including 17 active smokers. After topical anaesthesia, the 1.4- $\mathrm{mm}$ miniprobe was introduced into the bronchoscope working channel and advanced distally to the alveoli. Morphometric and cellular analyses were performed on selected frames harbouring a minimal compression effect.

In vivo acinar microimaging was obtained from each lung segment except for the apical and posterior segments of both upper lobes. Reproducible patterns, corresponding to the elastic framework of the axial and peripheral interstitial systems, were recorded from 192 separate acini. The mean \pm SD thickness of the acinar elastic fibres was $10 \pm 2.7 \mu \mathrm{m}$. Alveolar mouth diameters (mean \pm SD $278 \pm 53 \mu \mathrm{m}$ ) were normally distributed but appeared smaller in the right upper lobe and right medial basal segment. Lobular microvessels (median diameter $90 \mu \mathrm{m}$ ) were equally distributed throughout the lungs. Alveolar macrophages were not detectable in nonsmokers, whereas a specific tobacco-tar-induced fluorescence was observed in smoking subjects, providing fine details of the alveolar walls and macrophages. A strong correlation was found between the number of cigarettes smoked per day and the amount of large and mobile macrophages observed in vivo, as well as with the intensity of the macrophage alveolitis.

Fibred confocal fluorescence microscopy enables accurate exploration of the peripheral lung in vivo in both smokers and nonsmokers.

KEYWORDS: Bronchoscopy, diagnostic imaging, elastin, laser scanning confocal microscopy, pulmonary alveoli, tobacco smoking

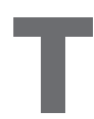
he external diameter of the thinnest available bronchoscope does not make it possible to image the respiratory tract in vivo beyond the bronchioles smaller than $3 \mathrm{~mm}$ in diameter [1]. Therefore, the pathology of the distal lung is currently assessed only in vitro, using techniques such as bronchoalveolar lavage and histology from transbronchial or open lung biopsies. No real-time imaging is available.

Fibred confocal fluorescence microscopy (FCFM) is a new technique based on the principle of confocal fluorescence microscopy [2, 3], where the microscope objective is replaced by a fibreoptic miniprobe, made of thousands of fibre cores. The miniprobe can be introduced into the 2-mm working channel of a flexible bronchoscope to provide in vivo endomicroscopic imaging of the human respiratory tract in real time [4].

In a previous study, FCFM was used to assess the autofluorescence microstructure of the proximal bronchial wall in vivo, and demonstrated that the main endogenous fluorophore that composes the FCFM signal is elastin [4]

Elastic fibres, which confer both extensibility and resilience to the pulmonary system, represent up to $50 \%$ of the lung connective tissue [5]. In the acinus, elastin is present in the axial backbone of the alveolar ducts and alveolar entrances, as well as in the external sheath of the extra-alveolar microvessels [5-8]. This axial system is interconnected to the peripheral interstitial system that
AFFILIATIONS

${ }^{*}$ Rouen University Hospital,

\#LITIS EA 4108 (groupe Quant-IF). Faculté de Médecine-Pharmacie, University of Rouen, Rouen, and -UMR CNRS 7033, Laboratoire de Biophysique Moléculaire, Cellulaire et Tissulaire, Université Pierre et Marie Curie - Paris 6, Paris, France.

\section{CORRESPONDENCE}

L. Thiberville, Clinique

Pneumologique, Hôpital Charles Nicolle, CHU de Rouen, 1 rue de Germont, 76031 Rouen Cedex,

France. Fax: 33232888240

E-mail: Luc.Thiberville@

univ-rouen.fr

Received:

June 032008

Accepted after revision:

December 172008

SUPPORT STATEMENT

This study was supported by the French Cancéropôle Nord-Ouest (Lille, France), the French Ministry of Health (Programme Hospitalier de Recherche Clinique 2007; Paris, France) and ADIR (Rouen, France). The unique sponsor of this study was the Rouen University Hospital

(Rouen), which designed the study and conducted the data collection, analysis and interpretation, and the writing of the report, independently of any commercial entity.

\section{STATEMENT OF INTEREST}

Statements of interest for $\mathrm{L}$. Thiberville and for the study itself can be found at www.erj.ersjournals.com/ misc/statements.dtl 
extends through the interlobular septa towards the pleura $[8,9]$. Following previous observations on the proximal bronchi, the current authors hypothesised that FCFM would be able to image the elastin framework microstructure of the distal lung in vivo [10].

The present study describes the use of FCFM to produce ex vivo and in vivo endomicroscopic images of the distal airways, from the distal bronchioles down to the alveolar ducts and sacs, in healthy smokers and nonsmokers. The main objective of the current study was to assess the possibility of obtaining reproducible, real-time FCFM in vivo imaging of the acinar structures in humans during bronchoscopy.

\section{METHODS}

\section{Ex vivo tissue samples}

A sample of a well preserved normal human lung stored in glutaraldehyde was kindly provided by E. Weibel (Institute of Anatomy, Bern, Switzerland). This sample had been fixed in situ shortly post mortem at a constant pressure of $20 \mathrm{cmH}_{2} \mathrm{O}$, as described [11]. After FCFM imaging, unstained sections of this sample were viewed using an epifluorescence microscope at $488 \mathrm{~nm}$ excitation (Leitz Aristoplan; Leica microsystems, Bensheim, Germany). Lobectomy samples were obtained in smoker and nonsmoker patients. Fresh sections were studied with FCFM first, using a micromanipulator to apply the probe onto the fresh tissue section, followed by histopathological analysis of the same tissue area.

\section{Subjects}

Smoker and nonsmoker healthy volunteers, selected from the Rouen Centre for Clinical Investigation (Rouen, France) filing system, were prospectively included in the clinical trial. The subjects had normal chest radiographs and respiratory function tests, and no history of respiratory, cardiac or bleeding disorders. The clinical study was approved by the Rouen University Ethical Committee (Rouen, France), and the Agence Française de Sécurité Sanitaire des Produits de Santé (SaintDenis, France). The subjects signed written informed consent before the procedure, and their participation was registered into the French National Database for healthy volunteers.

\section{In vivo alveolar explorations}

In vivo bronchoscopic procedures were performed in supine position on spontaneously breathing subjects, after topical anaesthesia of the airways. A 4.4-mm bronchoscope (MP60 model; Olympus, Tokyo, Japan) was inserted into the airways down to the smallest reachable bronchi. The FCFM miniprobe was then introduced into the working channel and gently advanced into the distal bronchiole until the alveolar system was observed. Up to eight bronchoalveolar areas were successively explored by selecting different bronchioles on one side of the bronchial tree (left or right lung) and the location of the corresponding lung segment was registered according to the international bronchial nomenclature [12]. Real-time alveolar images were continuously recorded during the procedure and stored for further analysis. Immediately after the alveoli were reached, the probe was slightly pulled back until the contact was lost, to ensure that the probe compression effect onto the alveolar system was minimal. A chest radiograph was performed before and after the procedure.

\section{FCFM imaging and spectroscopy system}

The FCFM Cellvizio ${ }^{\circledR}$-Lung spectroscope prototype used for the current clinical study was provided by Mauna Kea Technologies (Paris, France). The system used in the current study differed from the commercially available Cellvizio ${ }^{\circledR}$ Lung device (fig. 1) by the addition of a spectroscopic channel, which enabled the simultaneous recording of the spectrum and the microscopic images in the observed field of view [4]. The imaging system produced 9 frames $\cdot \mathrm{s}^{-1}$ for a $896 \times 640$ pixel image and a circular $600-\mu \mathrm{m}$ diameter field of view. The lateral resolution and depth of focus of the probe were $3.5 \mu \mathrm{m}$ and 0 $50 \mu \mathrm{m}$, respectively. A schematic diagram of the system, as well as the relative sizes of the probe tip and the distal lung structures, are shown in figure 1. Details of the system are provided in the online supplementary material.

\section{Image analysis}

For qualitative and quantitative assessment purposes, images of the intralobular structures were carefully selected from the last frames recorded before the contact was lost when pulling
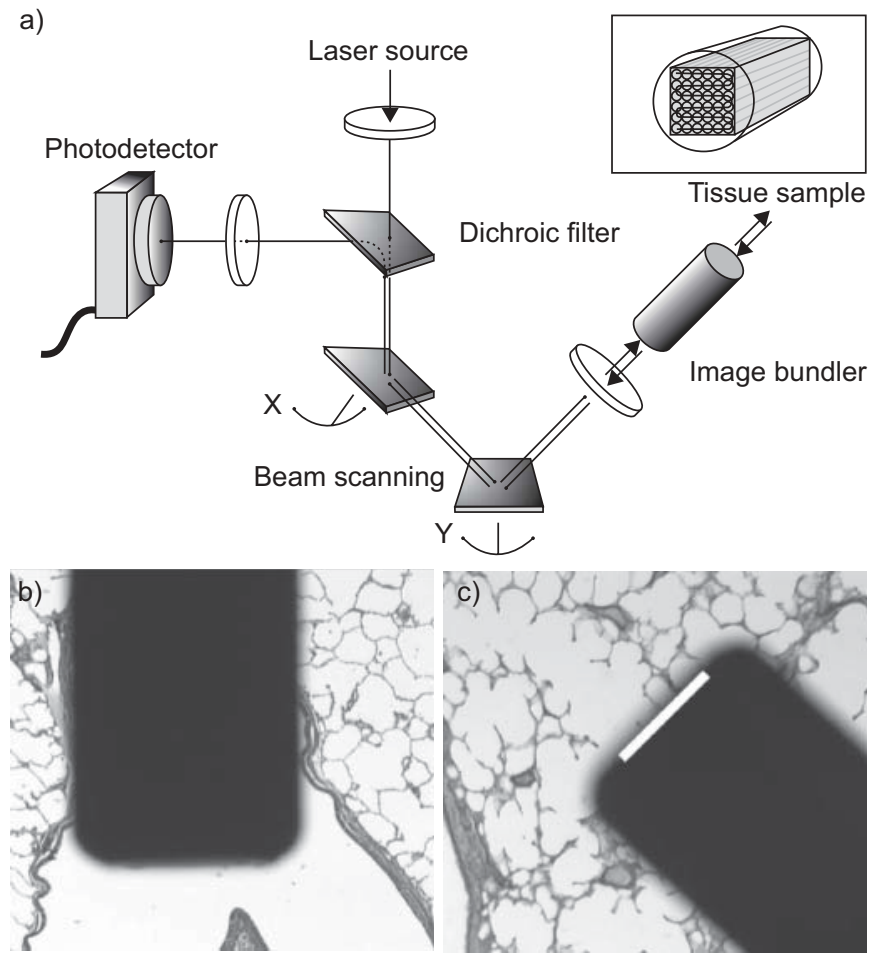

FIGURE 1. a) Diagram of the Cellvizio®-Lung System (Mauna Kea Technologies, Paris, France), and relative sizes of the 1.4-mm probe tip and the b) bronchiolar and c) alveolar structures. a) The fibred confocal fluorescence microscopy system is made of several components: a 1.4-mm miniaturised flexible fibred miniprobe made of thousands of fibre cores (upper right), a 488-nm excitation wavelength laser source, an XY scanning system (upper left), and a dedicated control and acquisition software that allows the reconstruction of dynamic images in real time. Note that the spectroscopy channel is not represented in the diagram; complete details of the spectroscopy system have been previously described [4]. b) The tip of the miniprobe is superimposed on a distal bronchiole lumen in a 7- $\mu \mathrm{m}$ fixed lung tissue section. c) The miniprobe is superimposed in front of an alveolar duct system. The white bar corresponds to the diameter of the fibre bundle $(600 \mu \mathrm{m})$ 
back the probe. This ensured that the deformation of the alveoli was minimal.

Morphometric analysis of the intra-acinar structures (i.e. thickness of the elastin fibres, alveolar ring entrance and microvessel diameters) as well as dynamic and cellular analyses (i.e. movement, size and number of alveolar macrophages per field of view) were separately performed by two observers (L. Thiberville and M. Salaün) using a dedicated software (MedViewer ${ }^{\circledR}$-1.1.1; Mauna Kea Technologies). The software allowed retrospective viewing of the data, image by image, as well as micrometric measurements of the fluorescent structures.

Fluorescence intensity was quantified using the MedViewer®'s Signal Quantification Toolbox, with the lower and upper level thresholds of the look-up table set to 1 and 8000, respectively. Fluorescence intensity was defined as the median of pixel intensities over the entire selected image. To take into account possible variations due to optical properties of the different miniprobes used in the study, in vivo tissue fluorescence intensity was normalised versus the fluorescence intensity of a known concentration of acriflavin solution $(1 / 1200 \mathrm{w} / \mathrm{v}$; Sigma Aldrich, St Louis, MO, USA). Results were also expressed as alveolar/bronchus fluorescence ratios, using a reference image from the proximal bronchus in each subject. This allowed intergroup comparisons (i.e. active smokers versus nonsmoking subjects) while taking into account intersubject fluorescence variability due to other causes, such as potential intrinsic variations in elastin fluorescence.

\section{Statistics}

Comparisons were performed using Chi-squared and MannWhitney tests, or ANOVA or Spearman's correlation test when required. Normality distribution was assessed using the Shapiro-Wilk test. Values of $\mathrm{p}<0.05$ were considered significant.

\section{RESULTS}

\section{Ex vivo FCFM of fixed and fresh human lung samples}

As can be seen in figure 2 and in the online supplementary video, the system resolution made it possible to image fine details of the capillary network in the alveolar walls (fig. 2a), the structure of the pleural lung surface (fig. 2b) and the epithelial
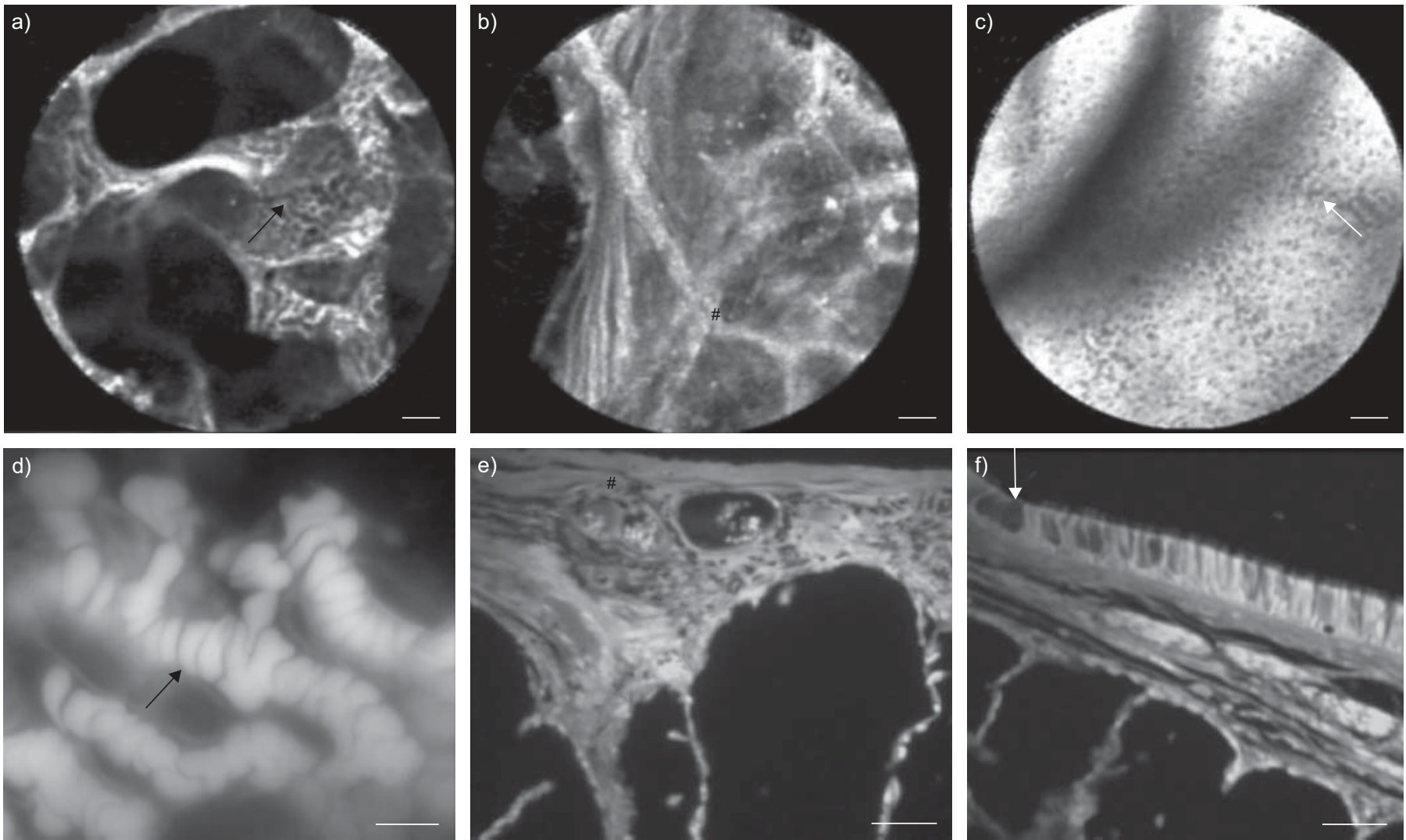

FIGURE 2. a-c) Fibred confocal fluorescence microscopy (FCFM) images of a well preserved lung sample fixed in glutaraldehyde at $20 \mathrm{cmH}_{2} \mathrm{O}$ pressure, and $\mathrm{d}-\mathrm{f}$ ) comparison with corresponding $10-\mu \mathrm{m}$ unstained histology sections, imaged using an oil immersion epifluorescence microscope. Both imaging modalities were performed at a 488-nm excitation wavelength. a) FCFM imaging of an alveolar duct system showing fine details of the capillary network on the alveolar walls (black arrow). Images were obtained after applying the probe onto the alveolated section of the sample. See online supplementary video for the dynamic sequences. b) FCFM image of the lung seen from the pleural surface. *: alveolar wall connecting to the peripheral connective tissue of the pleura. c) FCFM imaging from inside a bronchus showing an en face view of the epithelial layer. Goblet mucus cells appear as black spots (white arrow). d) Fluorescence microscopy of a 10- $\mu$ m thick section corresponding to the alveolar wall of a), showing details of fixative-induced fluorescent erythrocytes in the alveolar capillary network (black arrow). e) Fluorescence microscopy, pleural section and adjacent alveolar walls. f) Fluorescence microscopy of the bronchial section corresponding to (c). White arrow: goblet cells with cytoplasmic mucus appearing in black. Scale bars=a-c) $50 \mu \mathrm{m}$, d) $10 \mu \mathrm{m}$, e) $25 \mu \mathrm{m}$ and f) $20 \mu \mathrm{m}$. 
TABLE 1 In vivo alveolar endoscopy in smoking and nonsmoking healthy volunteers

\begin{tabular}{|c|c|c|c|}
\hline Subjects & 17 & 24 & 41 \\
\hline Male/female & $9 / 8$ & $10 / 14$ & $19 / 22$ \\
\hline FVC $\%$ predicted & $101 \pm 16$ & $104 \pm 15$ & $103 \pm 16$ \\
\hline Duration of alveolar procedure min & $14 \pm 6$ & $10 \pm 3$ & $11 \pm 5$ \\
\hline Elastic fibre thickness $\mu \mathrm{m}$ & $9.7 \pm 3.0$ & $10.4 \pm 2.3$ & $10 \pm 2.7$ \\
\hline Measurements & 445 & 464 & 909 \\
\hline Bronchus/Alexafluor fluorescence intensity ratio & $0.20 \pm 0.11$ & $0.12 \pm 0.07$ & $0.14 \pm 0.09$ \\
\hline Alveolus/bronchus fluorescence intensity ratio & $0.86 \pm 0.69^{\#}$ & $0.25 \pm 0.17^{\#}$ & \\
\hline Microvessel diameter $\mu \mathrm{m}$ & $95 \pm 47$ & $89 \pm 56$ & $90 \pm 50$ \\
\hline
\end{tabular}

Data are presented as $\mathrm{n}$ and mean $\pm \mathrm{SD}$, with the exception of microvessel diameter, which is presented as median \pm interquartile range. FVC: forced vital capacity

\#: $p<0.0001$, Mann-Whitney test; ${ }^{\circ}: p<0.0001$, Chi-squared test.

cell layer of the bronchial surface (fig. 2c). This imaging was possible because of the very strong autofluorescence of every structure in the sample, including erythrocytes, which was induced by the prolonged fixation time in glutaraldehyde. In contrast to this long-term fixed tissue imaging, FCFM of fresh lung tissue exclusively imaged the elastin component of the connective tissue of the distal lung based on its natural autofluorescence (online supplementary fig. 1)

\section{In vivo bronchoalveolar imaging during endoscopy of active smokers and nonsmoking subjects}

A total of 41 healthy subjects (19 males) were recruited, including 17 active smokers. Among nonsmoking subjects, 20 had never smoked and three were ex-smokers who had stopped smoking for 8,18 and 20 yrs. One subject was an occasional smoker ( 1 cigarette $\cdot$ month $\left.^{-1}\right)$ and was classified as a nonsmoking individual. This subject had not smoked for 2 months, but had been exposed to passive cigarette smoking 3 days before the procedure. The main characteristics of the subjects are indicated in table 1.

\section{Tolerance of the procedure}

The mean \pm SD duration of the alveolar imaging was $11 \pm 5$ min (table 1). The procedure was painless except for a few subjects who indicated a transient pain in regard to the lung region explored, indicating that the probe had reached the subpleural region. No bleeding was observed in the proximal bronchial tree. The chest radiograph performed after the procedure was normal in each case.

\section{In vivo distal bronchiolar imaging}

A total of 215 bronchiolar areas were explored (table 2). Due to the difficult bending of the bronchoscope when it contained the probe, the apical and posterior segment of both upper lobes (B1 and B2) could not be reached. Every other segment of both lungs was easily assessed during the procedures.
The endomicroscopic imaging of a distal bronchiole is illustrated in the online supplementary material (supplementary fig. 2 and online video). At this level, FCFM identified the helicoidal imprint of the peribronchiolar muscles on the inner surface of the bronchiole (online video). Due to the respective sizes of the probes and of the distal bronchiolar structures (fig. 1), the presence of alveolar buds in the respiratory bronchioles were identified in only two cases (supplementary fig. 2 and online video). The entry into the alveolar space was regularly obtained by penetration through the bronchiolar wall (online video).

\section{In vivo acinar FCFM imaging in nonsmoking individuals}

At the acinar level, FCFM in nonsmoking subjects exclusively imaged the elastin framework of the alveolar ducts, alveolar entrance rings and extra-alveolar microvessels, with a typical aspect displayed in figure 3 , figure 4 and the online video.

The in vivo aspects of the distal interstitial framework varied depending on the angle of penetration of the probe into the alveolar unit. A direct view down the axis of the duct or an oblique view were commonly obtained, from which the "helical" or "looped" shape of the duct's elastic structure could be easily recognised (fig. $3 a$ and c, and online video) [13]. In contrast, a lateral viewpoint could show the extension of the alveolar duct along its longitudinal axis, and the resulting elongation of the entrance rings in synchronisation with the respiration cycle (fig. $3 \mathrm{~b}$ and online video).

Figure 5 and the online video show the consequences of the probe tip compression onto the acinar structures in front of an alveolar duct. These images show that the compression enabled the visualisation of deeper structures down the axis of the duct, providing a 3D-like effect, without perceptible deformation of the structures in the first plane of focus. 


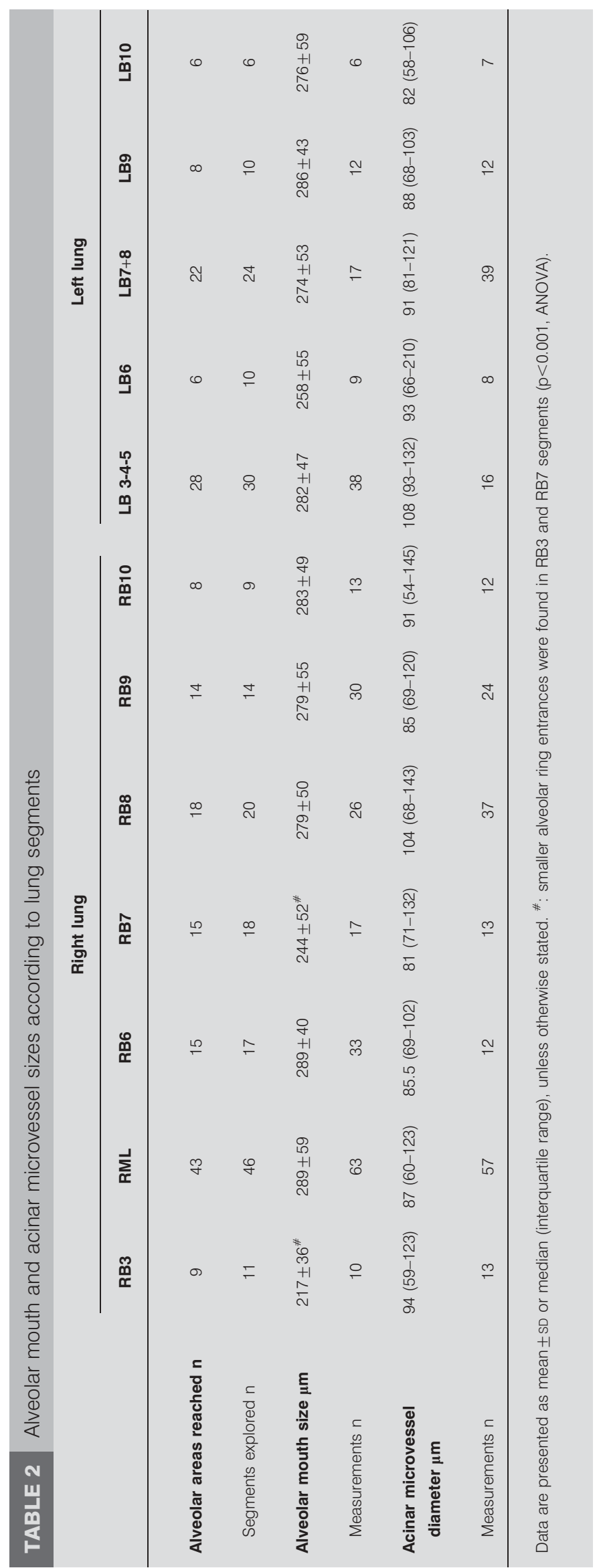

\section{Acinar fluorescent structure measurements and reproducibility}

Alveolar structures were reached in 192 out of the 215 bronchial segments explored (table 2). Figure 4 displays examples of alveolar mouth diameter measurements, performed on selected images with minimal compression effect. Tables 1 and 2 indicate the mean alveolar mouth diameters measured with this technique. Diameters from the 274 alveolar mouths assessed in vivo appeared normally distributed (Shapiro-Wilk test; fig. 4), with a mean \pm SD size of $278 \pm 53 \mu \mathrm{m}$ (table 1 ). The alveolar mouths appeared significantly smaller in the ventral segment of the right upper lobe (RB3) and in the right medial basal/paracardiac segment (RB7), compared with the other lung segments explored $(\mathrm{p}<0.001$, ANOVA; table 2$)$.

The mean \pm SD thickness of the elastic fibres was $10 \pm 2.7 \mu \mathrm{m}$ for the whole group of subjects (909 measurements; table 1).

Figure 4 and the online video (mosaicing reconstruction) [14] show the usual FCFM appearance of the lobular microvessels in vivo. A total of 250 different microvessels were identified (tables 1 and 2). In contrast to the alveolar entrances, microvessel diameters were not normally distributed due to the presence of rare large vessels (fig. 4). The median size of lobular microvessels was $90 \pm 50 \mu \mathrm{m}$ (table 1). They appeared equally distributed throughout the lungs (tables 1 and 2).

There was no significant difference in the alveolar mouth size or microvessel diameters according to age and sex of the subjects (data not shown).

\section{Alveolar imaging of active smokers}

Alveolar fluorescence imaging in smokers differed dramatically from imaging in nonsmokers (fig. 6). The alveolar entrances and alveolar ducts of smokers were usually filled with highly fluorescent cells, the movements of which could be clearly observed (see online video). Bronchoalveolar lavage analysis from smokers using fluorescence and conventional microscopy confirmed that these fluorescent cells correspond exclusively to alveolar macrophages [15].

Fluorescent macrophages were observed in every smoking subject except one cigar smoker (table 1). In contrast, there were no detectable fluorescent cells in the alveolar space of nonsmoking subjects, except for one subject heavily exposed to passive cigarette smoke 3 days before the procedure. Therefore, fluorescent cellular imaging appeared highly specific for smokers in healthy individuals ( $\mathrm{p}<0.0001$; table 1 ).

Due to specific contrast imaging in smokers, bubble-like structures were sometimes detectable on the alveolar surface (fig. $6 \mathrm{c}$ and online video). In addition, details of the alveolar wall could be identified in smokers, including recesses resembling the pores of Kohn, and fine longitudinal structures compatible with the surface of the capillary network (fig. 6d).

\section{Origin of the alveolar fluorescence in smoking and nonsmoking individuals}

Spectroscopic analysis of the fluorescence in smoking and nonsmoking individuals is displayed in figure 7. Figure $7 \mathrm{a}$ shows the normalised autofluorescence emission spectra obtained during in vivo alveoscopy from nonsmoking subjects 

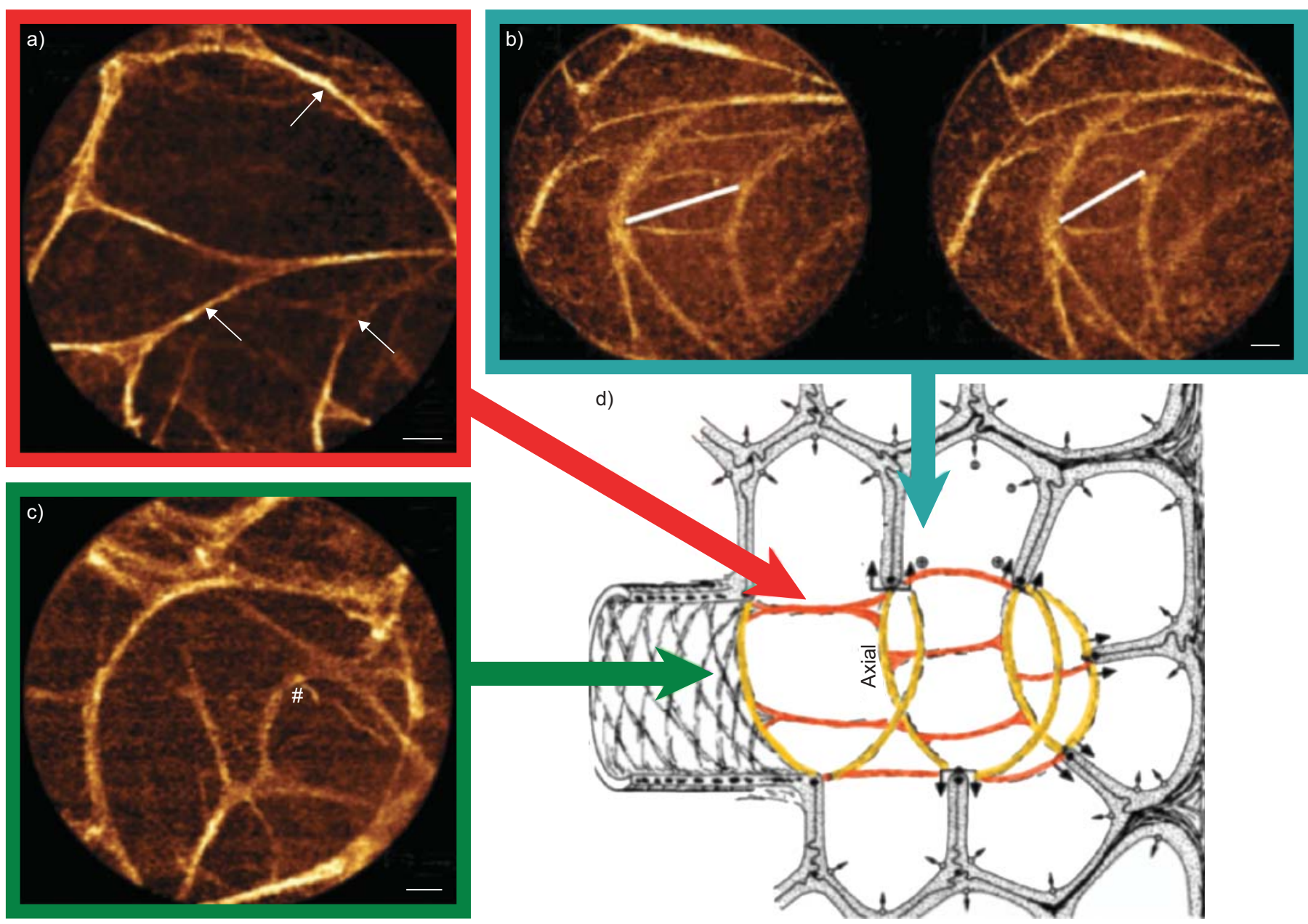

FIGURE 3. In vivo alveolar confocal fluorescence imaging according to the relative positions of the probe tip and the alveolar duct axis (nonsmoker healthy volunteers) $\mathrm{a}-\mathrm{c}$ ) The different points of view of the alveolar duct are indicated according to d) schematic Weibel's representation of the peripheral lung elastic backbone (modified from [8] with permission from the publisher). Thick coloured arrows: supposed angles of penetration of the miniprobe into the alveolar unit. In nonsmokers, fibred confocal fluorescence microscopy (FCFM) mainly imaged the elastin that is concentrated in the axial fibred system (represented as orange and yellow coloured lines in d)), which forms the edges of the alveolar duct rim region (yellow) and the alveolar ring entrances (orange). a) Oblique view of the duct axial fibred system, showing the helical or looped shape of the duct's edge (arrows). b) Lateral view of the axial system, showing how the elastin fibres at the alveolar openings are interconnected with the frame of the alveolar duct, in a "ladder-stitch" manner. Two sequential images show the elongation of the alveolar duct along its longitudinal axis in synchrony with the respiration (online video 7 ) White bars: size variations of the alveolar entrances. c) View down the axis of the duct, showing alveolar ring entrances in the back planes $(\#)$, due to a slight compression effect (see online video 6 for a dynamic sequence). Scale bars $=50 \mu \mathrm{m}$.

and from human lung-extracted elastin powder. Repeated experiments in several nonsmoking subjects clearly showed that the FCFM signal originated from the elastin backbone of the peripheral lung.

In contrast, figure $7 \mathrm{~b}$ shows spectra acquired from the distal filter of a smoked cigarette compared with in vivo alveolar autofluorescence spectra from several active smokers. Autofluorescence spectra from active smoker alveoli and from smoked tobacco tar were found to be similar, apart from the initial part of the alveoli curve corresponding to the elastin component.

A significant variation in the intensity of the autofluorescence signal was observed between the subjects in relation to their age, the oldest individuals presenting the strongest signal $(\mathrm{r}=0.61, \mathrm{p}=0.0036$; Spearman's correlation test).
Fluorescence intensity values for proximal bronchi and alveoli are indicated in table 1 . Whereas proximal bronchus fluorescence intensity appeared similar in smoking and nonsmoking individuals, the alveolus/bronchus intensity ratio was found to be significantly higher in current smokers $(p<0.001$, Mann-Whitney test). In active smokers, the alveolus/bronchus fluorescence ratio also appeared correlated with the number of alveolar macrophages per field of view $(\mathrm{r}=0.50, \mathrm{p}<0.001$; Spearman's test; fig. $8 b$ ).

Table 1 displays the intersubject fluorescence variability (expressed as the standard deviation of the alveolus/bronchus fluorescence intensity ratio). Fluorescence variability was higher in active smokers compared with nonsmoking subjects, in relation to the intensity of the tobacco-induced macrophage alveolitis ( $p<0.001$, Fisher's variance comparison test). 

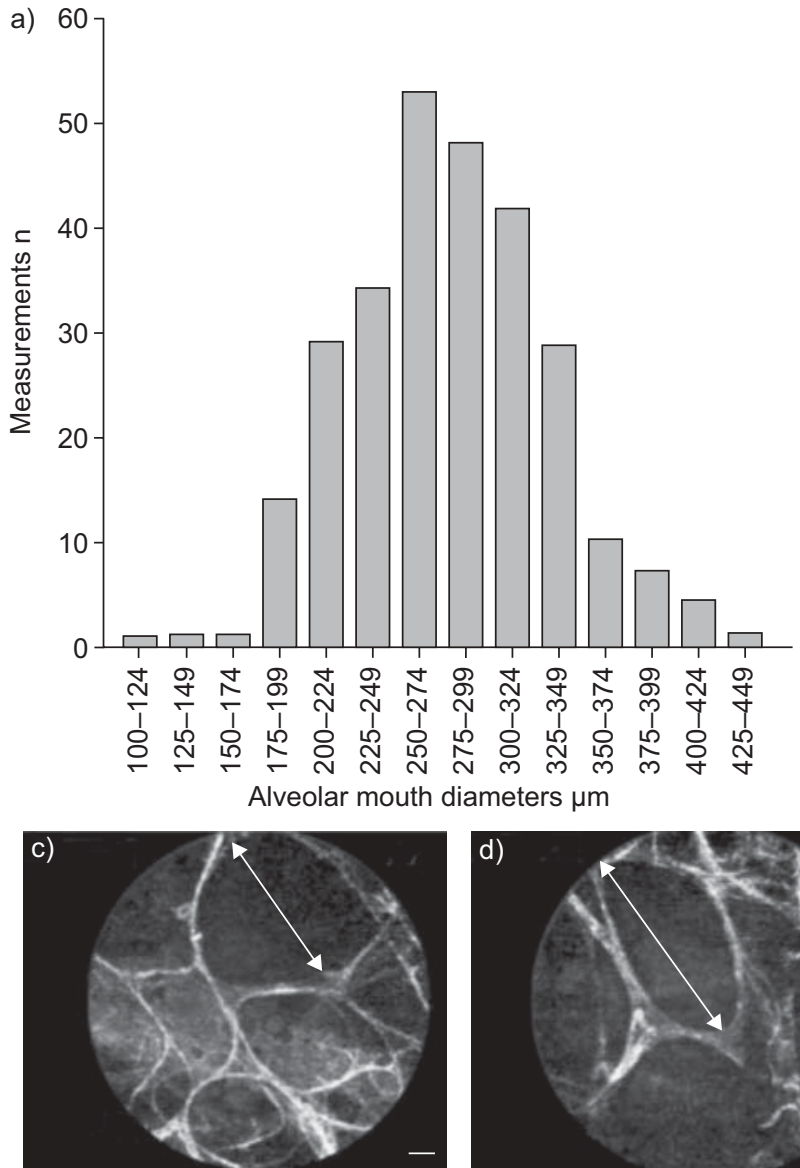
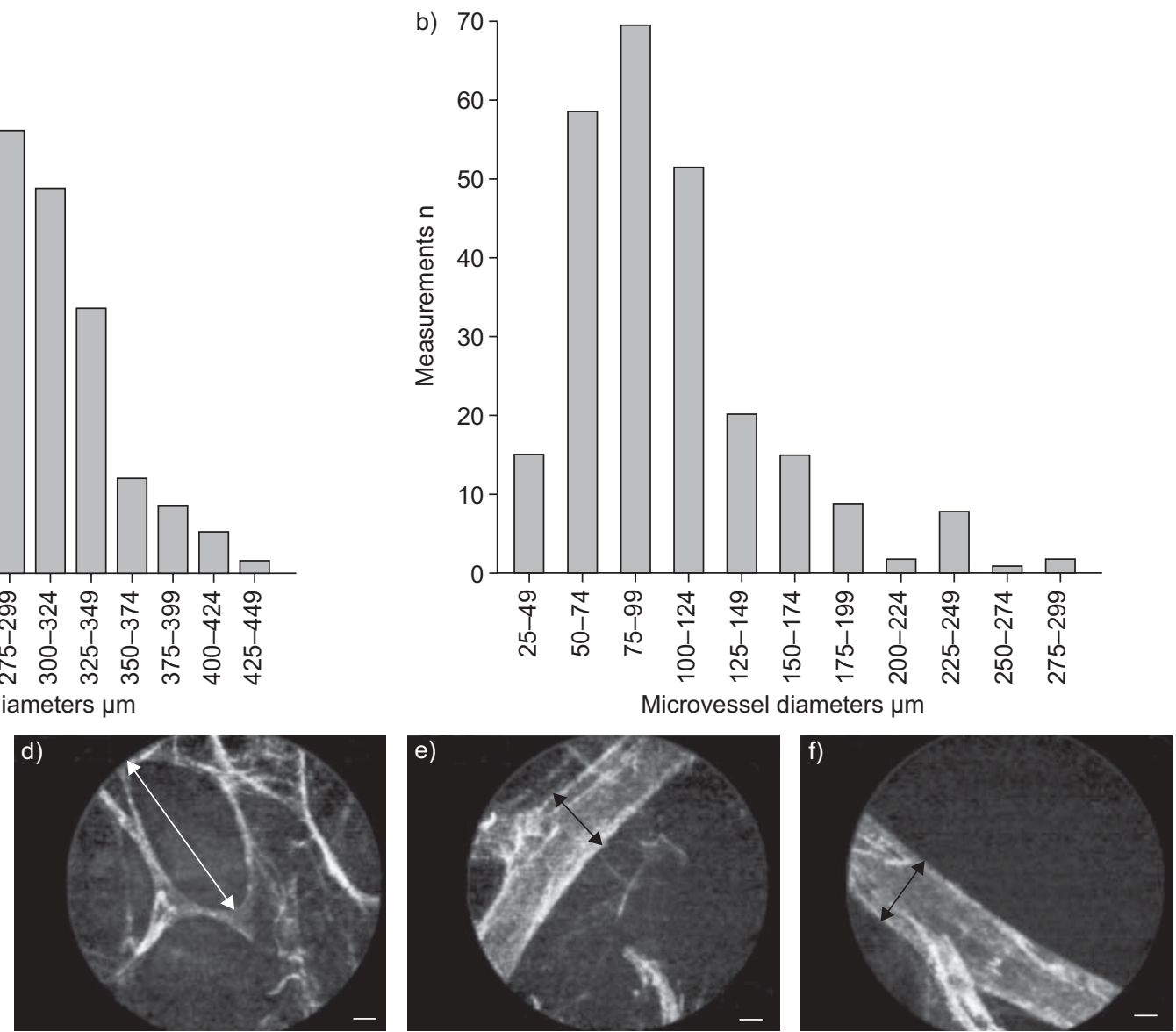

FIGURE 4. Alveolar mouth ( $a, c$ and d) and lobular microvessel (b, e and f) measurements. Distribution of a) alveolar mouth and b) lobular microvessel diameters. $c$ and d) Examples of alveolar ring measurements. White arrows: size of the entrance ring at minimal compression. e and f) Examples of extra-alveolar microvessel measurements. Black arrows: diameters measured upstream of the vessel bifurcation. Scale bars $=50 \mu \mathrm{m}$
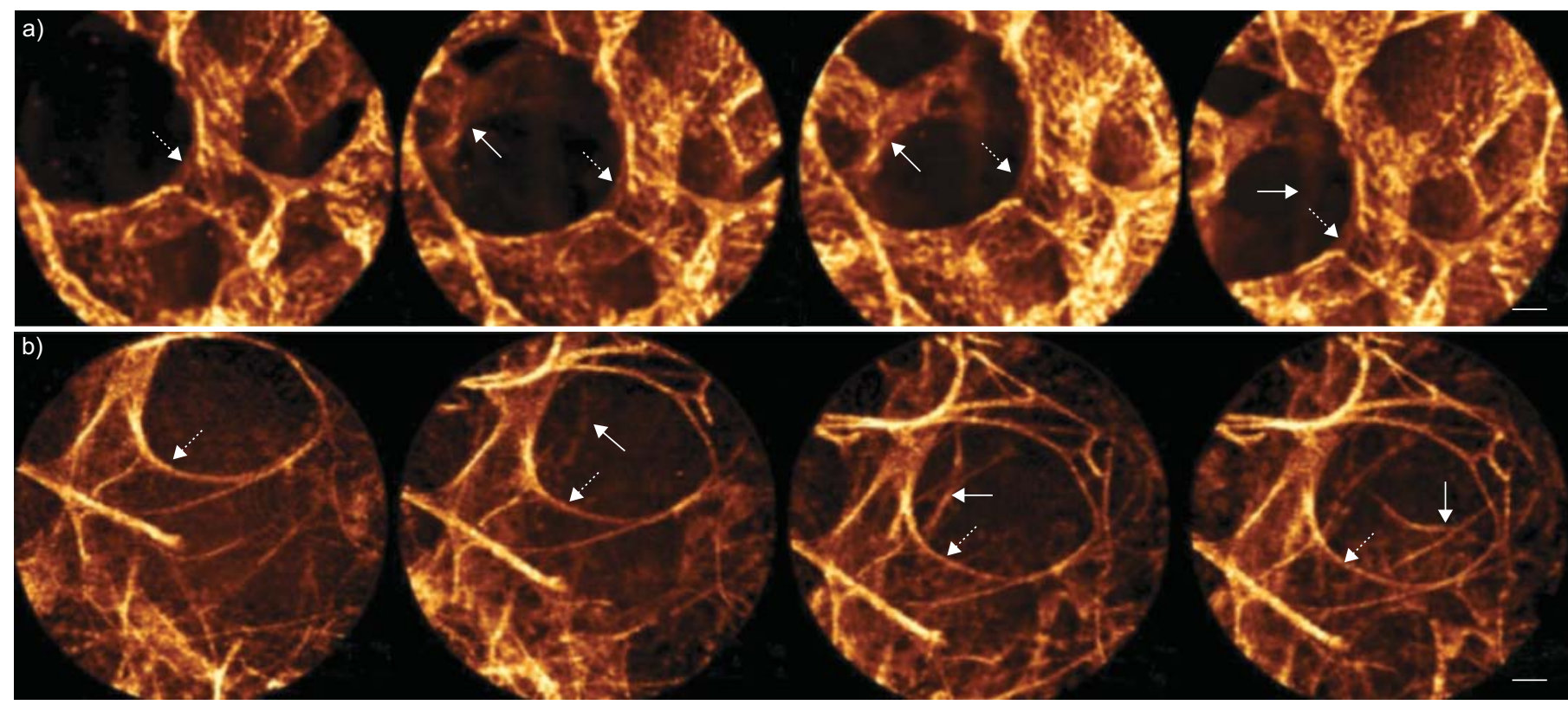

FIGURE 5. a) Ex vivo and b) in vivo consequences of a probe tip compression applied on the lung tissue in the axis of the alveolar duct. From left to right: effect of increasing pressure on the tissue (see online video for the dynamic sequence). a) Ex vivo fixed sample. b) In vivo fibred confocal fluorescence microscopy images from a nonsmoking individual. Dashed arrows: edges of the alveolar duct; arrows: successive appearance of distant alveolar duct structures. Compression allowed for visualisation of deeper structures down the axis of the duct, providing a 3D-like effect, without perceptible deformation in the first plane of focus. Scale bars $=50 \mu \mathrm{m}$. 

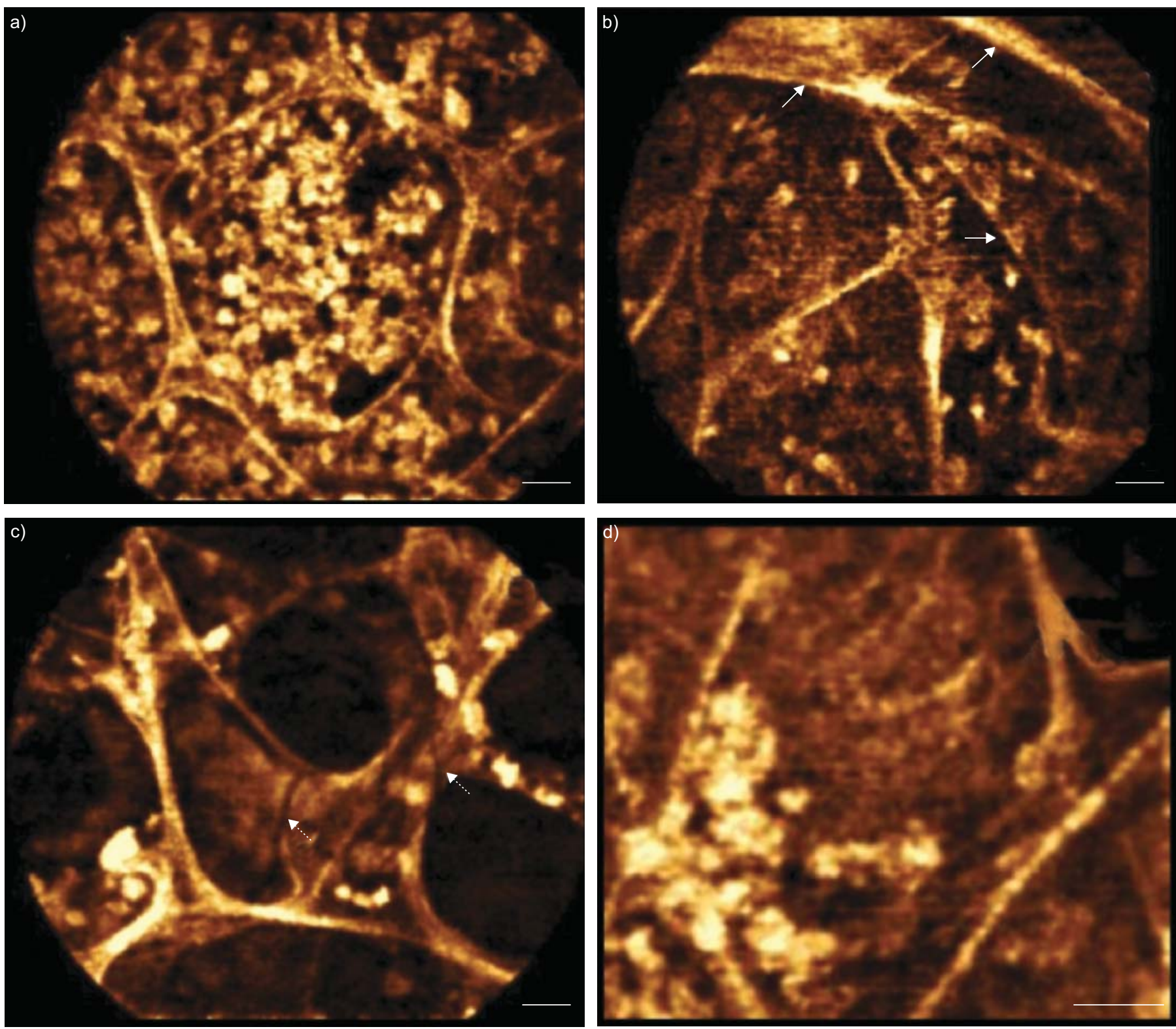

FIGURE 6. In vivo fibred confocal fluorescence microscopy alveolar imaging in smokers. a) Alveolar system filled with highly fluorescent alveolar macrophages. Axia view down the alveolar duct. The corresponding image in a nonsmoker can be found in figure 3c. Dynamic imaging can be seen in online video 9. b) Alveolar duct, oblique view. Arrows: helical arrangement of the axial cables of the alveolar duct. The corresponding image in a nonsmoker can be found in figure $3 a$. c) In vivo imaging of the alveolar walls, showing bubbles on the surface of the alveolar wall and alveolar entrance (dashed arrows; see online video). d) Fine details of the alveolar wall from the same patient as in a). Lower left: fluorescent alveolar macrophages; upper right: triple lines of alveolar wall at the junction of three alveoli; middle: linear structures that may correspond to pleats of alveolar wall or to the relief of the capillary within the alveolar space. Dynamic imaging can be visualised at the end of the online video sequence. Scale bars $=50 \mu \mathrm{m}$

Intra-individual variability appeared lower than inter-individual variability in nonsmoking subjects ( 0.095 versus 0.17 , respectively).

\section{In vivo cellular imaging in smoking subjects}

Figure 8 illustrates how in vivo FCFM could assess the alveolar macrophage infiltration in smokers. As expected, the amount of cigarettes smoked per day was correlated with the total number of fluorescent macrophages per field of view ( $r=0.70, p<0.0001$; Spearman's correlation test, fig. 8a), as well as with the mean number of macrophages larger than $30 \mu \mathrm{m}$ per explored alveolar area $(r=0.77, p=0.0002$; fig. $8 \mathrm{c})$. Subjects presenting mobile alveolar macrophages smoked a significantly greater number of cigarettes a day, compared with patients who harboured immobile alveolar macrophages on FCFM imaging (mean \pm SD $16.5 \pm 4.35$ versus $6.75 \pm 4.08$ cigarettes $\cdot$ day $^{-1} ; \mathrm{p}=0.0019$, Mann-Whitney test; fig. $8 \mathrm{~d})$.

\section{DISCUSSION}

Ventilation and gas exchange can be altered by a large variety of diffuse parenchymal lung diseases (DPLD), for which diagnosis can be approached by using high-resolution computed tomography and nonimaging endoscopic procedures $[16,17]$. 

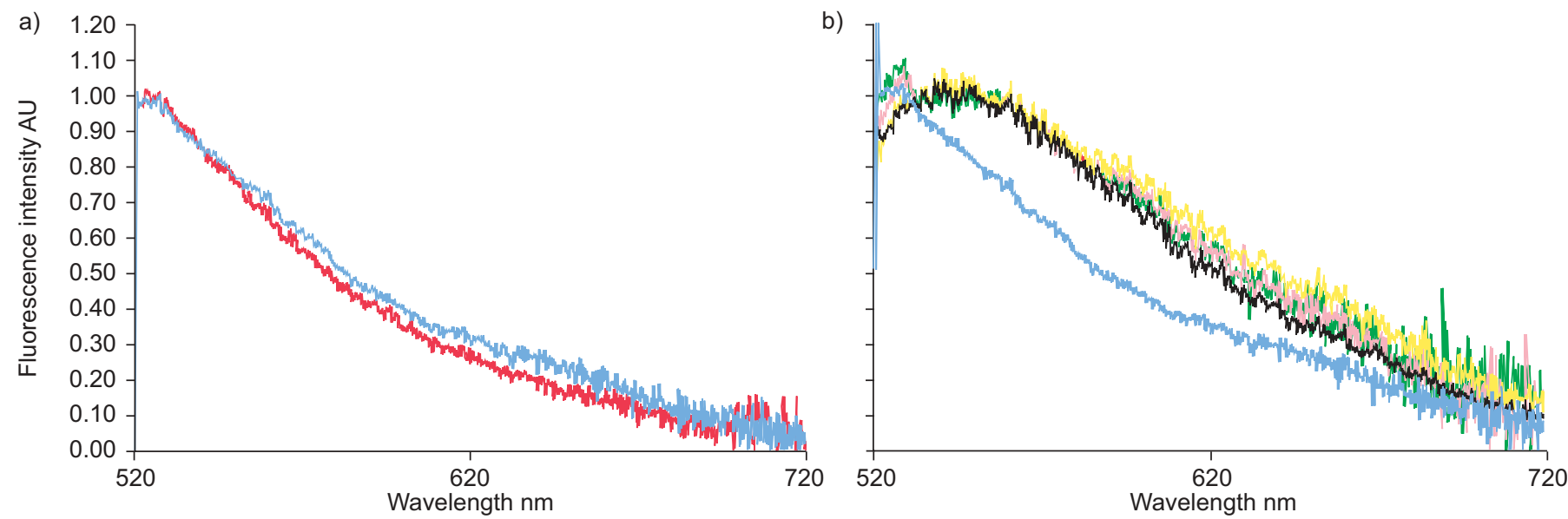

FIGURE 7. Autofluorescence emission spectra of alveolar systems from a) nonsmokers and b) healthy smokers, during in vivo alveoscopy. Blue: elastin powder; red: nonsmoker alveoli; green, pink, yellow: smoker alveoli; black: smoked cigarette. AU: arbitrary units.
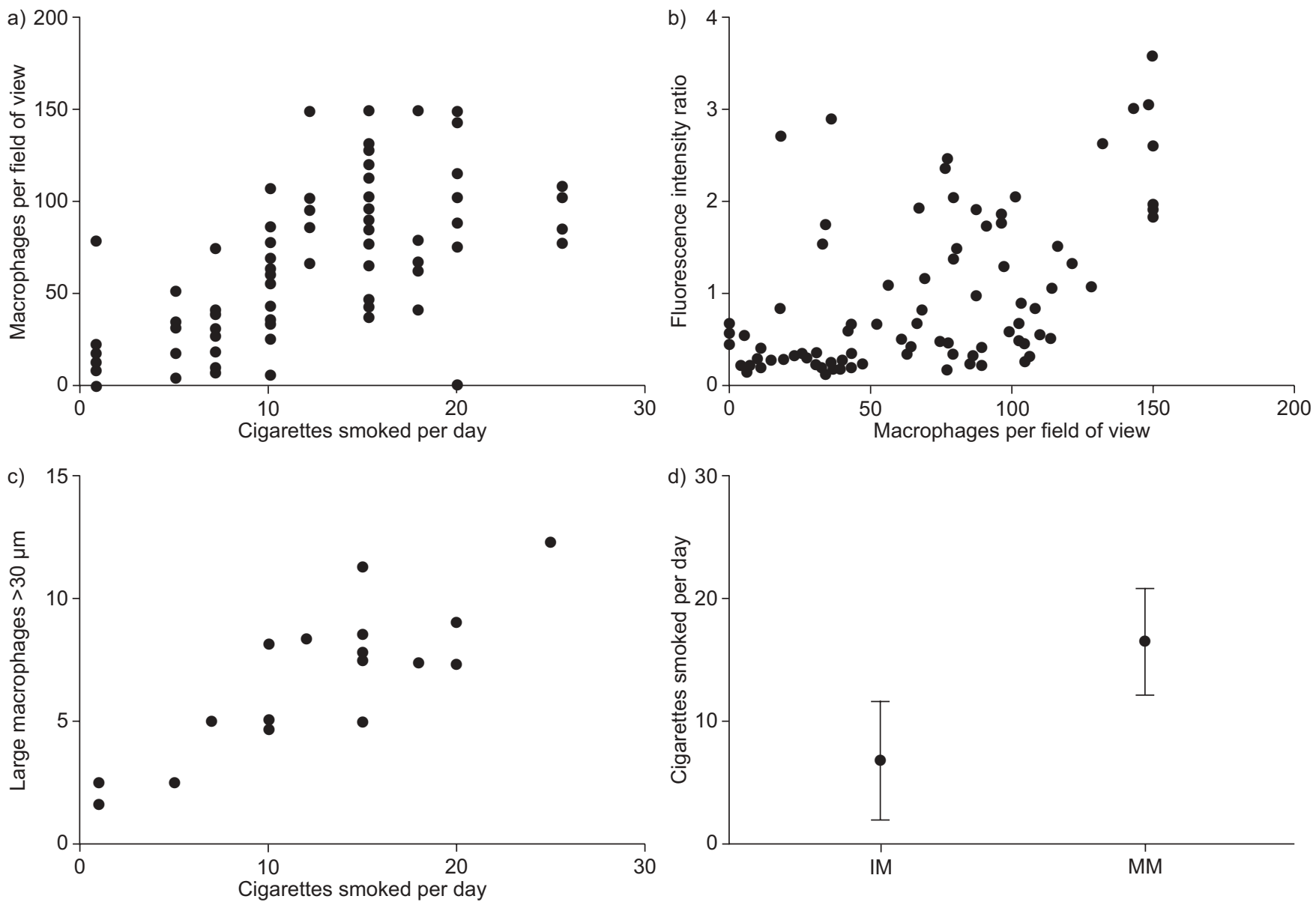

FIGURE 8. Fibred confocal fluorescence microscopy (FCFM) cellular imaging in smoking individuals. a) In vivo FCFM imaging of macrophage alveolitis. Correlation between the number of cigarettes smoked per day and the number of fluorescent macrophages observed per field of view $(r=0.70, p<0.0001$; Spearman's correlation test). b) Alveolus/bronchus fluorescence intensity ratio. Alveolar fluorescence intensity appeared linked to the intensity of the macrophage alveolitis $(r=0.50, p<0.0001$ Spearman's correlation test). c) Large activated macrophages in smokers. Correlation between the number of cigarettes smoked per day and the mean number of macrophages $>30 \mu \mathrm{m}$ per explored alveolar area ( $r=0.77, p=0.0002$; Spearman's correlation test). d) In vivo FCFM imaging of macrophage mobility. The number of cigarettes smoked per day appeared higher in subjects presenting mobile alveolar macrophages (MM; mean \pm SD $16.5 \pm 4.35$ cigarettes $\cdot$ day $^{-1}$ ) compared with those with immobile macrophages (IM; $6.75 \pm 4.08$ cigarettes $\cdot$ day $^{-1} ; \mathrm{p}=0.0019$; Mann-Whitney test). Data are presented as mean $\pm \mathrm{SD}$. 
In many cases, the diagnosis of DPLD needs a surgical lung biopsy, with multiple sampling from different lobes [18-21]. These procedures are not devoid of morbidity, and cannot usually be repeated on the same patient, despite the possibility of histopathological variations over time [22-24]. In this context, the development of an in vivo, minimally invasive method of peripheral lung microscopic imaging appears greatly needed.

The broncho-alveoscopy procedure described in the present study is the first technique that makes it possible to obtain realtime, high-resolution, microstructural images of lobular and alveolar lung structures in living humans.

In the current series of subjects, it has been shown that the technique is remarkably well tolerated in topically anaesthetised spontaneously breathing subjects. Due to the lack of pain receptors in the bronchial tree down to the subpleural level, the penetration of the probe into the pulmonary lobule through the distal bronchiolar wall is painless. In addition, acinar imaging was not associated with detectable bleeding in the proximal airways, in contrast to what is usually observed with transbronchial biopsy sampling. This can be explained by the small blood flow in the thin nourishing vessels of the bronchiolar wall, by the low pressure in the alveolar capillaries that could be altered during the progression of the probe, as well as by the smooth design of the probe tip that can displace the extra-alveolar microvessels without damage. Finally, no pleural complication was observed, despite multiple lung segment imaging during the endoscopy. Therefore, fluorescence broncho-alveoscopy appears to be a minimally invasive procedure, which could be repeated over time on the same subject.

The elastin network imaging obtained by this in vivo technique appears very similar to what is observed using delicate methods of lung preservation and specific elastin staining $e x$ vivo [5-7]. In the present study, reproducible images of the elastic fibres that encircle the alveolar openings, reinforce the alveolar rim region of the alveolar ducts and surround the extra-alveolar microvessels were obtained. After careful selection of images corresponding to minimally compressed structures, it was found that alveolar opening sizes were normally distributed in the whole series of subjects, with mean values compatible with what is observed using unbiased stereological methods in vitro $[25,26]$. In the current in vivo series, variations in alveolar mouth diameters also accounted for the modifications of the alveolar duct size with respiration. This may explain the fact that smaller alveolar mouths were observed in right B3 and B7 segments, presumably related to the lower ventilation of these segments in the supine position. The technique also permitted precise measurements of the lobular microvessels, which appeared equally but not normally distributed throughout the lungs, due to the observation of rarer larger vessels. More information on these structures may be available in the future by using intravenous injection of fluorescein solution, whose distribution kinetics should make it possible to differentiate precapillary arterioles from the post capillary vessels.

Some technical issues should be addressed with respect to the interpretation of the distal lung FCFM imaging. First, because of the orthogonal branching and the small calibre of the terminal and respiratory bronchioles in humans compared to the probe size, alveolar imaging regularly bypassed the respiratory bronchioles, which were identified in only two cases in the current series by the observation of alveolar buds branching on their walls. This could represent a limitation for the study of the distal membranous and respiratory bronchioles [27]. This difficulty may be solved in the future by using thinner probes, such as the ones used for small animal imaging [28]. During the procedure, the probe had to progress through the bronchiolar wall and peribronchiolar connective tissue before stabilising in the acinus. A direct view down the axis of the alveolar duct could often be obtained (see online video), presumably because the resistance of the elastic structures to the probe progression is higher at the centre of the axial fibred system, where the concentration of collagen and elastin is maximal [5]. However, images of the alveolar duct from other perspectives could also be observed, which are more difficult to interpret but may provide other important information, such as the degree of extensibility of the alveolar duct along its axis.

Secondly, the probe progression into the lobule supposes the disruption of alveolar walls, followed by a compression effect on the more resistant ductal structures. Minimal imaging distortion was observed when the probe was applied on the axis of the duct, resulting in the visualisation of more details in the background planes than supposed by the $50-\mu \mathrm{m}$ depth of focus of the system [28]. By approaching the first plane of focus toward the backplanes, this produced the illusion of an increasing working distance, with a resulting 3D optical effect. Although the compression to the alveolar structures appeared more delicate to control in vivo, it was found that the best results could be obtained by gently pulling back the probe once the alveolar imaging was obtained and analysing the last images before the contact was lost. This technique provided reproducible imaging and measurement of the alveolar structures.

Thirdly, fluorescence microimaging has some advantages and limitations that should be underlined. In contrast to reflectance imaging, fluorescence microimaging is devoid of interference with the reflected and refracted light at the air-liquid interface, because the backscattered excitation light is filtered out by the detection system. This merely produces a small decrease in the signal intensity at the air-liquid interface, without other optical artefact. In the present study, this property allowed the visualisation of bubble-like structures as well as of the presence of fluorescent cells within this liquid phase. These observations indicated that a small quantity of liquid interposes at the probe tip that may contribute to the imaging signal. The nature of this liquid interface could not be identified from the current observations, but could be related to a small amount of bronchial lining fluid taken along during the progression of the probe, or to the surfactant that covers the alveolar walls.

Conversely, FCFM exclusively records the signal coming from fluorescent structures in response to appropriate excitation wavelengths. Although in vivo FCFM in nonsmokers only imaged the elastin of the peripheral and axial connective tissues, data from the literature indicate that such information might be 
helpful for the exploration of several pathological conditions. Qualitative and quantitative modifications of the alveolar elastin network have been demonstrated ex vivo in usual interstitial pneumonitis and in emphysema [29, 30]. Elastin alterations have also been observed in lung adenocarcinomas, which correspond to intratumoural foci of active fibroblast proliferation associated with a bad prognosis of the tumour [31, 32]. Therefore, the technique might prove to be helpful in a large variety of diseases, including interstitial lung diseases.

In this series of healthy volunteers, the only detectable cells were alveolar macrophages containing tobacco tar. This clearly differentiated smokers from nonsmokers on the basis of FCFM images, and indicated that the tobacco tar acts as an exogenous fluorophore that participates in the specific alveolar imaging. In the current study it was shown that FCFM could precisely assess early acinar tobacco-induced changes, such as macrophage number, size and mobility, indicating that the technique could be applied to the exploration of pulmonary diseases in cigarette smokers [33].

The simplified view of the acinar structures observed in vivo in nonsmoking subjects contrasted with the detailed imaging of the alveolar capillary network and fluorescent erythrocytes obtained from the fixed human lung sample. Such detailed imaging had been observed by others on fixed samples using conventional confocal fluorescence microscopy [30]. To the current authors' knowledge, this is related to modifications of the tissue fluorescence induced by the long-term aldehyde fixation [34,35]. This observation confirms that the nature of the endogenous fluorophores represents the critical factor for FCFM imaging, more than the resolution capabilities of the system. Future evolutions of the technique appear feasible by using combinations of various wavelengths [36], which could make it possible to image several fluorophores of the lung connective network simultaneously, such as collagen species and elastin. Moreover, studies in patients may be able to observe other fluorescent cells that are not normally visible in healthy subjects.

In conclusion, fluorescence broncho-alveoscopy is a minimally invasive and safe technique that can be conducted in spontaneously breathing subjects. The current study represents the initial step towards defining in vivo fibred confocal fluorescence microscopy alveolar imaging of the normal human lung. This description will be useful for the study of specific fibred confocal fluorescence microscopy abnormalities in peripheral lung diseases. Such abnormalities could then be tested as diagnostic indicators and easily monitored to test the efficacy of therapeutic intervention.

\section{ACKNOWLEDGEMENTS}

The authors are indebted to E. Weibel and M. Ochs at the Institute for Anatomy in Bern, Switzerland, for providing them with a very well preserved and well fixed human lung sample, as well as for their very helpful comments on the interpretation of the in vivo FCFM alveolar imaging. The help of the Rouen University Centre for Clinical Investigation (Rouen, France) in recruiting healthy volunteers was greatly appreciated.

\section{REFERENCES}

1 Shinagawa N, Yamazaki K, Onodera Y, et al. Factors related to diagnostic sensitivity using an ultrathin bronchoscope under CT guidance. Chest 2007; 131: 549-553.

2 Le Goualher G, Perchant A, Genet M, et al. Towards optical biopsies with an integrated fibered confocal fluorescence microscope. In: Barillot C, Haynor DR, Hellier P, eds. Medical Image Computing and Computer-Assisted Intervention - MICCAI 2004. Lecture Notes in Computer Science. Berlin/Heidelberg, Springer, 2004; 3217: 761-768.

3 MacAulay C, Lane P, Richards-Kortum R. In vivo pathology: microendoscopy as a new endoscopic imaging modality. Gastrointest Endosc Clin N Am 2004; 14: 595-620.

4 Thiberville L, Moreno-Swirc S, Vercauteren T, Peltier E, Cavé C, Bourg-Heckly G. In vivo imaging of the bronchial wall microstructure using fibered confocal fluorescence microscopy. Am J Respir Crit Care Med 2007; 175: 22-31.

5 Mercer RR, Crapo JD. Spatial distribution of collagen and elastin fibers in the lungs. J Appl Physiol 1990; 69: 756-765.

6 Gonçalves CA, Figueiredo MH, Bairos VA. Three-dimensional organization of the elastic fibres in the rat lung. Anat Rec 1995; 243: 63-70.

7 Toshima M, Ohtani Y, Ohtani O. Three-dimensional architecture of elastin and collagen fiber networks in the human and rat lung. Arch Histol Cytol 2004; 67: 31-40.

8 Weibel ER, Sapoval B, Filoche M. Design of peripheral airways for efficient gas exchange. Respir Physiol Neurobiol 2005; 148: 3-21.

9 Weibel ER. How to make an alveolus. Eur Respir J 2008; 31: 483-485.

10 Thiberville L, Bourg-Heckly G, Peltier E, Cave C. Perendoscopic alveolar imaging using fluorescent confocal fibered microscopy. Eur Respir J 2006; 28: Suppl. 50, 155s-156s.

11 Gehr P, Bachofen M, Weibel ER. The normal human lung: ultrastructure and morphometric estimation of diffusion capacity. Respir Physiol 1978; 32: 121-140.

12 Prakash UB, Fontana RS. Functional classification of bronchial carinae. Chest 1984; 86: 770-772.

13 Cookson MJ, Davies CJ, Entwistle A, Whimster WF. The microanatomy of the alveolar duct of the human lung imaged by confocal microscopy and visualised with computer-based 3D reconstruction. Comput Med Imaging Graph 1993; 17: 201-210.

14 Vercauteren T, Perchant A, Malandain G, Pennec X, Ayache N. Robust mosaicing with correction of motion distortions and tissue deformations for in vivo fibered microscopy. Med Image Anal 2006; 10: 673-692.

15 Pauly JL, Allison EM, Hurley EL, Nwogu CE, Wallace PK, Paszkiewicz GM. Fluorescent human lung macrophages analyzed by spectral confocal laser scanning microscopy and multispectral cytometry. Microsc Res Tech 2005; 67: 79-89.

16 Schwarz MI, King TE Jr. Approach to the evaluation and diagnosis of interstitial lung diseases. In: King TE Jr, Schwarz MI, eds. Interstitial Lung Diseases. 4th Edn. Hamilton, BC Decker 2003; pp. 1-30.

17 Aziz ZA, Wells AU, Hansell DM, et al. HRCT diagnosis of diffuse parenchymal lung disease: inter-observer variation. Thorax 2004; 59: 506-511.

$18 \mathrm{du}$ Bois R, King TE Jr. Challenges in pulmonary fibrosis. 5: The NSIP/UIP debate. Thorax 2007; 62: 1008-1012. 
19 Wells AU. Histopathologic diagnosis in diffuse lung disease: an ailing gold standard. Am J Respir Crit Care Med 2004; 170: 828-829.

20 Monaghan H, Wells AU, Colby TV, du Bois RM, Hansell DM, Nicholson AG. Prognostic implications of histologic patterns in multiple surgical lung biopsies from patients with idiopathic interstitial pneumonias. Chest 2004; 125: 522-526.

21 Nicholson AG, Addis BJ, Bharucha H, et al. Inter-observer variation between pathologists in diffuse parenchymal lung disease. Thorax 2004; 59: 500-505.

22 Kreider ME, Hansen-Flaschen J, Ahmad NN, et al. Complications of video-assisted thoracoscopic lung biopsy in patients with interstitial lung disease. Ann Thorac Surg 2007; 83: 1140-1144.

23 Misumi S, Lynch DA. Idiopathic pulmonary fibrosis/usual interstitial pneumonia: imaging diagnosis, spectrum of abnormalities, and temporal progression. Proc Am Thorac Soc 2006; 3: 307-314.

24 Silva CI, Müller NL, Hansell DM, Lee KS, Nicholson AG, Wells AU. Nonspecific interstitial pneumonia and idiopathic pulmonary fibrosis: changes in pattern and distribution of disease over time. Radiology 2008; 247: 251-259.

25 Weibel ER, Hsia CC, Ochs M. How much is there really? Why stereology is essential in lung morphometry. J Appl Physiol 2007; 102: 459-467.

26 Hyde DM, Tyler NK, Putney LF, Singh P, Gundersen HJ. Total number and mean size of alveoli in mammalian lung estimated using fractionator sampling and unbiased estimates of the Euler characteristic of alveolar openings. Anat Rec A Discov Mol Cell Evol Biol 2004; 277: 216-226.

27 Verbeken EK, Cauberghs M, Lauweryns JM, van de Woestijne KP. Anatomy of membranous bronchioles in normal, senile and emphysematous human lungs. I Appl Physiol 1994; 77: 1875-1884.

28 Vincent P, Maskos U, Charvet I, et al. Live imaging of neural structure and function by fibred fluorescence microscopy. EMBO Rep 2006; 7: 1154-1161.

29 Honda $\mathrm{T}$, Ota $\mathrm{H}$, Arai $\mathrm{K}$, et al. Three-dimensional analysis of alveolar structure in usual interstitial pneumonia. Virchows Arch 2002; 441: 47-52.

30 Black PN, Ching PS, Beaumont B, Ranasinghe S, Taylor G, Merrilees MJ. Changes in elastic fibres in the small airways and alveoli in COPD. Eur Respir J 2008; 31: 998-1004.

31 Eto T, Suzuki H, Honda A, Nagashima Y. The changes of the stromal elastotic framework in the growth of peripheral lung adenocarcinomas. Cancer 1996; 77: 646-656.

32 Honda T, Ota $\mathrm{H}$, Sano $\mathrm{K}$, et al. Alveolar shrinkage in bronchioloalveolar carcinoma without central fibrosis. Lung Cancer 2002; 36: 283-288.

33 Wells AU, Nicholson AG, Hansell DM. Challenges in pulmonary fibrosis. 4: Smoking-induced diffuse interstitial lung diseases. Thorax 2007; 62: 904-910.

34 Abe M, Takahashi M, Horiuchi K, Nagano A. The changes in crosslink contents in tissues after formalin fixation. Anal Biochem 2003; 318: 118-123.

35 Gabrecht T, Andrejevic-Blant S, Wagnières G. Blue-violet excited autofluorescence spectroscopy and imaging of normal and cancerous human bronchial tissue after formalin fixation. Photochem Photobiol 2007; 83: 450-458.

36 Jean F, Bourg-Heckly G, Viellerobe B. Fibered confocal spectroscopy and multicolor imaging system for in vivo fluorescence analysis. Opt Express 2007; 15: 4008-4017. 